\title{
Noninvasive quantification of myocardial blood flow and coronary flow reserve
}

\author{
George A. Beller, MD
}

\section{INTRODUCTION}

The four papers in this symposium were presented at the 2009 Annual Scientific Session of the American Society of Nuclear Cardiology. In recent years, great advances have been made in the noninvasive evaluation of myocardial blood flow, particularly with positron emission tomography employing dynamic imaging for measuring absolute blood flow and flow reserve. Other techniques such as cardiac computed tomography (CT) and cardiac magnetic resonance imaging (CMR) are being exploited for their potential for quantitating blood flow. Better quantitation of regional myocardial blood flow will enhance the detection of multivessel disease, particularly in instances where conventional SPECT imaging shows uniform tracer uptake in the presence of balanced ischemia and diffuse abnormal flow reserve. Additionally, measurement of flow reserve with vasodilator stress permits identification of abnormal physiological states that affect the coronary microcirculation where no high grade coronary artery stenoses are present. Early coronary atherosclerosis or just the presence of multiple coronary artery disease risk factors is also often associated with endothelial and microvascular dysfunction that can now be adequately assessed noninvasively with quantitative imaging of flow reserve.

The first paper by Heinrich Schelbert, entitled Anatomy and Physiology of Coronary Blood Flow provides a superb and comprehensive review of coronary flow physiology including the determinants of myocardial blood flow, the mechanisms of alterations in coronary circulatory function in disease, the role of the microcirculation and the manner in which structural and functional alterations of the coronary circulation can be distinguished. It forms a perfect background for the rationale of quantitative blood flow imaging discussed in the other four papers. The second paper by Klein, Beanlands and deKemp, entitled, Quantification of
Myocardial Blood Flow and Flow Reserve-Technical Aspects, provides an excellent detailed review of the technical aspects of measuring myocardial blood flow using PET imaging instrumentation and software.

The third paper by Rob Beanlands, entitled, The Clinical Utility of Assessing Myocardial Blood Flow Using Positron Emission Tomography, clearly demonstrates the enhanced accuracy of detecting CAD and determining its extent using PET. He critically reviews the data in the literature that show the ability to detect impaired myocardial blood flow before significant epicardial coronary artery stenoses develop, and how the traditional cardiovascular risk factors may contribute to abnormal flow reserve in response to vasodilator stress. Clinical data indicating that PET can identify microvascular dysfunction is persuasive as is the enhanced diagnostic and prognostic value of quantitative PET over other nuclear imaging techniques that only assess relative differences in myocardial tracer uptake. The final paper in the symposium by Albert Lardo, entitled, Assessment of Coronary Blood Flow With Computed Tomography and Magnetic Resonance Imaging, reviews the emerging data on how myocardial blood flow and flow reserve can be measured using first-pass perfusion imaging with CT and CMR technology. The strengths and limitations of these technologies are superbly discussed and the first clinical studies are very well summarized.

In summary, these four papers provide a state-of-the art review of the noninvasive quantification of myocardial blood flow and why such an approach enhances our diagnostic and prognostic capabilities. In the future, with new imaging agents and advances in imaging systems and computer software, quantitative imaging may become the preferred approach to evaluating symptomatic and preclinical CAD.

\footnotetext{
The papers in this symposium were derived from presentations made by the authors at the 14th Annual Scientific Session of the American Society of Nuclear Cardiology, October 1-4, 2009, in Minneapolis, Minnesota.

J Nucl Cardiol 2010;17:544.

$1071-3581 / \$ 34.00$

Copyright $(2010$ by the American Society of Nuclear Cardiology. doi:10.1007/s12350-010-9261-z
} 